\title{
A Complete Workflow for Subnanometer Resolution Subtomogram Averaging In Situ
}

Steven Ludtke and Muyuan Chen

Baylor College of Medicine, Houston, Texas, United States

Subtomogram averaging provides capabilities normal CryoEM single particle reconstruction does not. First, it permits study of highly flexible assemblies in which the single view provided by single particle analysis produces ambiguous results. More importantly, however, it offers the ability to solve structures directly within the intact (or cryo-milled) cell. It is only by combining reductionist in vitro structural studies with in situ studies that a complete picture of macromolecular function can emerge. Unfortunately past studies in a cellular context were often limited to resolutions in the 20-40 $\AA$ range, and required complex sequences of multiple software packages to achieve acceptable results. Recent work has enabled subtomogram averaging in which single particle reconstruction methods are hybridized with traditional 3D subtomogram alignment and averaging approaches to produce high resolution structures while retaining the advantages provided by imaging each field of view over a range of angles.

We have developed a complete workflow within EMAN2, spanning the entire process from raw tilt series to high resolution subtomogram averaging using PPPT (per particle per tilt) alignment and image correction, without need for any other software, and with automatic bookkeeping at all stages of the process. Key features of this pipeline include: a novel, fully automated tilt series alignment algorithm, which works with or without fiducials, and is successful on virtually every tilt series; tomogram reconstruction using a tiled Fourier approach which rivals the quality of SIRT, but with the speed of backprojection; deep-learning based 3-D particle picking, with support for multiple particle classes; automatic initial model generation using stochastic gradient methods; per-tilt geometry aware CTF fitting; and, finally, per-particle per-tilt alignment and correction to produce high resolution subtomogram averages. In addition to providing improved results with standard EMPIAR data sets for in-vitro tomography, we have also solved multiple structures from cellular data to subnanometer resolution using this technique. We will present an overview of the methodology as well as a representative high resolution result solved using this method. We will also discuss alterations to typical tomography imaging parameters to achieve more optimal results at high resolution in cells.

\section{References}

Chen, M., Bell, J.M., Shi, X. et al. A complete data processing workflow for cryo-ET and subtomogram averaging. Nat Methods 16, 1161-1168 (2019) 